Gazi University
Journal of Science
http://dergipark.gov.tr/gujs

\title{
A New Mixture of Exponential-Gamma Distribution
}

\author{
Nosakhare EKHOSUEHI ${ }^{(D)}$, Lawrence Chukwudumebi NZEI*(D) , Festus OPONE \\ Department of Mathematics, University of Benin, Benin City, Nigeria
}

\section{Highlights}

- This paper present a new mixture probability distribution.

- The aim of the proposed distribution is to present a more flexible model for lifetime data.

- Some important properties of the proposed distribution are studied.

- Simulation study is carried out to examine the accuracy of the MLE for different sample sizes.

- The usefulness of this distribution is examined with some real lifetime data from literature.

\section{Article Info}

Received: $26 / 10 / 2018$

Accepted: $24 / 07 / 2019$

\section{Keywords}

Gamma Mixture

Structural properties

Standardized Lindley

Maximum Likelihood

Estimation

\begin{abstract}
A new distribution called New Mixture of Exponential-Gamma Distribution is presented in this paper. This new distribution contains exponential and standardized Lindley distributions as sub models. Some of the structural properties of the proposed distribution which include the survival function, hazard rate function, moments, moment generating function, quantile function, distribution of order statistics and Renyi entropy are obtained. The maximum likelihood method of estimation was used to estimate the parameters of the distribution. A Simulation study was carried out to examine the performance and accuracy of the maximum likelihood estimates of the proposed distribution. An application of the proposed distribution to two real lifetime datasets is presented to illustrate its usefulness and superiority over some existing related models.
\end{abstract}

\section{INTRODUCTION}

In statistics, modeling and analyzing lifetime data have become very vital because of its application to real life data in many fields such as engineering, biomedical, social sciences, finance amongst others. To this effect, many classical distributions have been used to fit these kind of data sets. Some of these classical distributions include but not limited to the Exponential, Weibull, Gamma, Rayleigh distributions and their generalizations: See (Gupta and Kundu, [1]) for more details. Here we consider the popular gamma distribution for analyzing real life data. A random variable $Y$ is from the gamma distribution with shape parameter $\beta$ and rate parameter $\lambda$ if the probability density function (pdf) and cumulative distribution function $(\mathrm{CDF})$ are respectively given by

$$
f(y ; \beta, \lambda)=\frac{\lambda(\lambda y)^{\beta-1}}{\Gamma(\beta)} e^{-\lambda y} ; \quad y>0, \beta, \lambda>0
$$

and

$$
F(y ; \beta, \lambda)=\frac{\gamma(\beta, \lambda y)}{\Gamma(\beta)} ; \quad y>0, \lambda, \beta>0
$$


where $\gamma(\alpha, y)=\int_{0}^{y} t^{\alpha-1} e^{-t} d t$ is the lower incomplete gamma function and the corresponding upper incomplete gamma is written as $\Gamma(\alpha, y)=\int_{y}^{\infty} t^{\alpha-1} e^{-t} d t$. It can be shown that $\Gamma(\alpha)=\Gamma(\alpha, y)+\gamma(\alpha, y)$

Again, exponential distribution which is a special case of the gamma distribution when the shape parameter $\beta=1$, is a popular classical probability distribution used for analysing real life data.

Let $Y$ be a random variable from exponential distribution with respective pdf and CDF as

$$
f(y, \lambda)=\lambda e^{-\lambda y} ; y>0, \lambda>0
$$

and

$$
F(y, \lambda)=1-e^{-\lambda y} ; y>0, \lambda>0
$$

However, generalizations of the gamma distribution have been introduced by many authors: (Nadarajah and Gupta, [2]) introduced a generalized gamma distribution with application to drought data. A generalization of the gamma distribution was also introduced by (Mahdi and Gupta, [3]), (Stacy, [4]) introduced a new generalization of the gamma distribution, (Elbatal et al., [5]) introduced a new generalized Lindley distribution and (Gayan and Mavis, [6]) presented a generalized power Lindley distribution with application to cancer patients data, (Samir et al., [7]) proposed the log-gamma-Rayleigh distribution. Other related literature can be found in $[8-15]$ and many others.

The concept of mixture distributions is to produce a new distribution which should have more flexible behaviour compared to the based (mixed) distributions. It is one of the most crucial ways to obtain new probability distributions in applied probability used in several research areas. Sattayatham and Talangtam [16], introduced the finite mixture lognormal distributions for fitting of motor insurance claims data. Satsayamon, et al. [17] presented a new family of generalized gamma distribution as a mixture of the generalized gamma and length biased generalized gamma distributions.

This study is motivated as a result of lack of published work in the mixture of gamma distribution family with mini-modal shape in any literature to the best our knowledge. Mini-modal in this contest is used to describe a curve with one minimal point and one maximal point. Thus we present a lifetime model called "New Mixture of Exponential-Gamma (NMEG) Distribution". The main idea is to choose the mixing proportion in terms of the existing parameters such that a more flexible distribution is generated. The new distribution include as special cases the exponential, gamma and standardized Lindley distributions. We investigate its properties and demonstrate that this new distribution can serve as an alternative model to some existing classical distributions.

\section{THE NEW MIXTURE OF EXPONENTIAL-GAMMA DISTRIBUTION}

Let $f_{1}(y), f_{2}(y)$ be the pdfs and $F_{1}(y), F_{2}(y)$ be the CDFs of exponential and gamma distributions respectively of a random variable $\mathrm{Y}$, then the pdf and $\mathrm{CDF}$ of the proposed distribution of random variable $\mathrm{Y}$ are respectively of the form: 


$$
f(y)=w f_{1}(y)+(1-w) f_{2}(y)
$$

and

$$
F(y)=w F_{1}(y)+(1-w) F_{2}(y)
$$

where $0 \leq w \leq 1$ such that $w=\frac{1}{1+\beta}$, is called the mixing proportion.

Using (1) and (3) in (5), the pdf of the proposed mixture distribution is obtained as follows

$$
\begin{aligned}
f(y ; \beta, \lambda) & =\frac{1}{1+\beta} \lambda e^{-\lambda y}+\frac{\beta}{1+\beta} \frac{\lambda(\lambda y)^{\beta-1}}{\Gamma(\beta)} e^{-\lambda y}, \\
& =\frac{\lambda}{1+\beta}\left\{1+\frac{\beta(\lambda y)^{\beta-1}}{\Gamma(\beta)}\right\} e^{-\lambda y} ; y>0, \lambda>0, \beta>0
\end{aligned}
$$

and the corresponding CDF is obtained by substituting (2) and (4) into (6) as

$$
\begin{aligned}
F(y, \beta, \lambda) & =\frac{1}{1+\beta}\left(1-e^{-\lambda y)+\frac{\beta}{1+\beta}\left(\frac{\gamma(\beta, \lambda y)}{\Gamma(\beta)}\right),}\right. \\
& =\frac{1}{1+\beta}\left\{1-e^{-\lambda y}+\frac{\beta \gamma(\beta, \lambda y)}{\Gamma(\beta)}\right\} \quad y>0, \lambda>0, \beta>0 .
\end{aligned}
$$

The distribution with pdf (7) and CDF (8) is called the "New Mixture of Exponential-Gamma (NMEG)" distribution.

\subsection{Sub-Model of The NMEG Distribution}

The NMEG distribution includes some existing distributions as special cases:

1) If the shape parameter $\beta=1$, then the NMEG reduces to exponential distribution with pdf

$$
f_{E}(y ; \lambda)=\lambda e^{-\lambda y} ; y>0, \lambda>0 \text {. }
$$

2) If $\lambda=1$, the NMEG reduces to standardized Lindley distribution with pdf

$$
f_{S L}(y)=\frac{1}{1+\beta}\left(1+\frac{\beta y^{\beta-1}}{\Gamma(\beta)}\right) e^{-y} ; y>0, \beta>0 .
$$

3) If $w=0$, NMEG distribution reduces gamma distribution with pdf

$$
f_{G}(y)=\frac{\lambda(\lambda y)^{\beta-1}}{\Gamma(\beta)} ; y>0, \beta, \lambda>0 .
$$

\subsection{The Shape of NMEG Density Function}

The shape of the NMEG distribution pdf in (7) is discussed from Theorem 1.

Theorem 1. The density function, $f(y)$ of the NMEG distribution accommodates the following shapes:

(i) Decreasing when $0<\beta \leq 1, \lambda>0$ for all $y>0$, 
(ii) Unimodal when $1<\beta \leq 2, \lambda>0$ for all $y>0$,

(iii) Decreasing-Increasing-Decreasing when $\beta>2, \lambda>0$ for all $y>0$.

\section{Proof.}

We can study the behavior of $f(y)$ by considering the behavior of $\eta^{\prime}(y)$ and $\eta^{\prime \prime}(y)$ where $\eta(y)=\ln f(y)$, as follows:

$$
\begin{aligned}
& \eta(y)=\ln f(y)=\ln \left[\frac{\lambda}{(1+\beta) \Gamma(\beta)}\right]+\ln \left[\Gamma(\beta)+\beta \lambda^{\beta-1} y^{\beta-1}\right]-\lambda y \\
& \eta^{\prime}(y)=\frac{\beta(\beta-1) \lambda^{\beta-1} y^{\beta-2}}{\Gamma(\beta)+\beta \lambda^{\beta-1} y^{\beta-1}}-\lambda \\
& \eta^{\prime \prime}(y)=\frac{\left[\Gamma(\beta)+\beta \lambda^{\beta-1} y^{\beta-1}\right] \beta(\beta-1)(\beta-2) \lambda^{\beta-1} y^{\beta-3}-\left[\beta(\beta-1) \lambda^{\beta-1} y^{\beta-2}\right]^{2}}{\left[\Gamma(\beta)+\beta \lambda^{\beta-1} y-1\right]^{2}}
\end{aligned}
$$

(i) If $0<\beta \leq 1$ and $\lambda>0, \eta^{\prime}(y)<0$ for all $y>0$. This simply implies monotonic decreasing function.

(ii) If $1<\beta \leq 2$ and $\lambda>0, \eta^{\prime \prime}(y)<0$ for all $y>0$. This means that $f(y)$ has a maximum point (Mode) which is $\frac{1}{2 \lambda}$.

(iii) This is considered from the alternating signs of $\eta^{\prime}(y)$ defined in (9). For $\beta>2$, the sign of $\eta^{\prime}(y)$ changes from negative to positive and then back to negative as $y$ increases. This shows that the pdf is Decreasing-Increasing-Decreasing.

Figure 1 is the plot of the pdf of NMEG distribution for some values of parameters to visualize Theorem 1 above.

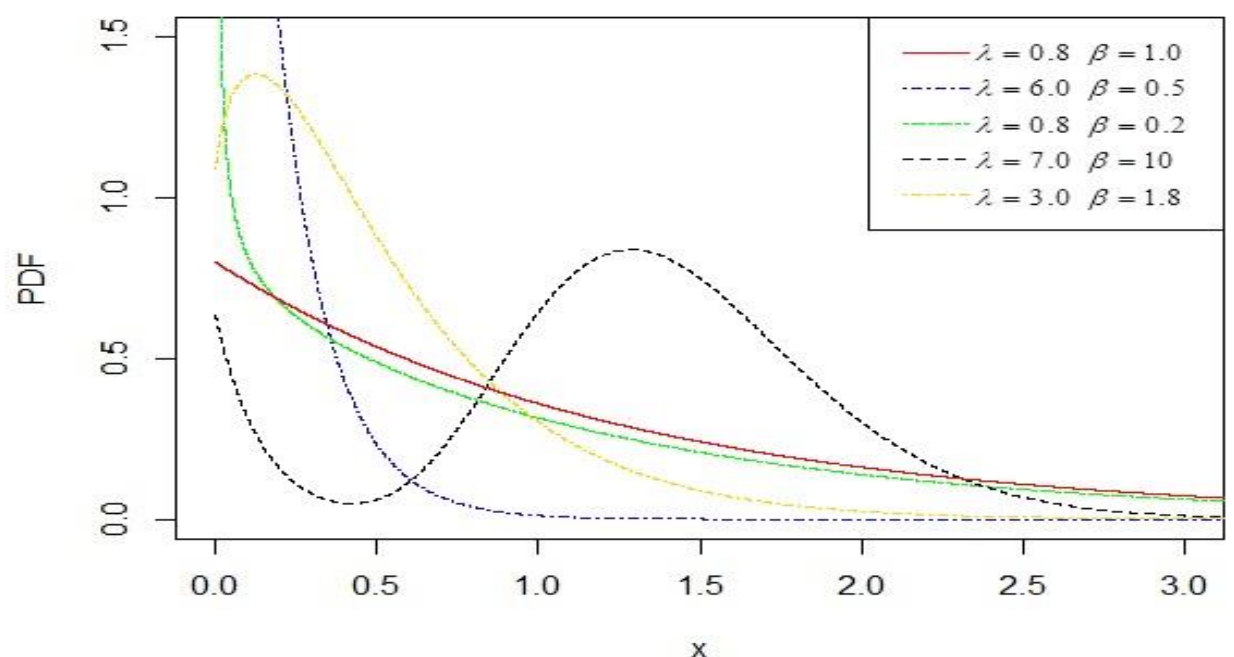

Figure 1. Thepdf of NMEG distribution for different 


\subsection{The Survival and Hazard Rate Function}

The survival (Reliability) and the hazard rate functions of the NMEG distribution are defined respectively as

$$
\bar{F}(y ; \lambda, \beta)=1-F(y ; \lambda, \beta)=1-\frac{1}{1+\beta}\left\{1+\frac{\beta \gamma(\beta, \lambda y)}{\Gamma(\beta)}-e^{-\lambda y}\right\}
$$

and

$$
h_{r}(y ; \lambda, \beta)=\frac{f(y)}{1-F(y)}=\frac{\lambda\left\{1+\frac{\beta(\lambda y)^{\beta-1}}{\Gamma(\beta)}\right\} e^{-\lambda y}}{1+\beta-\left\{1+\frac{\beta \gamma(\beta, \lambda y)}{\Gamma(\beta)}-e^{-\lambda y}\right\}} .
$$

\subsection{The Shape of NMEG Hazard Rate Function}

The shape of the NMEG distribution hazard rate functions in (12) is discussed from Theorem 2.

Theorem 2. The shape of the hazard rate function of the NMEG distribution can be summarized as follows:

(i) Constant for $\beta=1$, for all $\lambda, y>0$,

(ii) Decreasing when $\beta<1$, for all $\lambda, y>0$,

(iii) Increasing when $1<\beta<3$, for all $\lambda, y>0$,

(iv) Bathtub if $\beta \geq 3, \lambda>1$ for all $y>0$.

\section{Proof.}

To study the shape of the hazard rate function, it is sufficient to examine the behavior of $\phi(y)$ defined in Glasser [18] as

$$
\begin{aligned}
\phi(y) & =-\frac{f^{\prime}(y)}{f(y)}=-\frac{d}{d y} \ln [f(y)] \\
& =-\eta^{\prime}(y) .
\end{aligned}
$$

Then

$$
\phi^{\prime}(y)=-\eta^{\prime \prime}(y)
$$

where $\eta^{\prime \prime}(y)$ are defined in (10). Using Theorem (a) and Lemma (b) pg.668-670 in Glasser [18], it follows that:

(i) At $\beta=1$ and $\lambda>0, \phi^{\prime}(y)=0$ (Constant). Then the hazard rate function is constant for all $y>0$.

(ii) If $\beta<1$ and $\lambda>0$, we can clearly see that $\eta^{\prime \prime}(y)>0$ for all $y>0$ implies that $\phi^{\prime}(y)<0$. Hence, the hazard rate function is decreasing.

(iii) If $1<\beta<3$, for all $\lambda, y>0$, we have $\eta^{\prime \prime}(y)<0$ for all $y>0$ implies that $\phi^{\prime}(y)>0$, this means increasing hazard rate function. 
(iv) Following the condition stated in Glasser [18], $\eta^{\prime \prime}(y)<0$ changes its sign from negative to positive as $y$ increases with $\beta \geq 3, \lambda>1$. Then the hazard rate function is said to be bathtub shaped with minimal point at $y=\frac{1}{\lambda}\left(1+\frac{\sqrt{3}}{3}\right)$.

Figure 2 is the plot of the hazard rate function NMEG distribution for some values of parameters to visualiz Theorem 2 above.

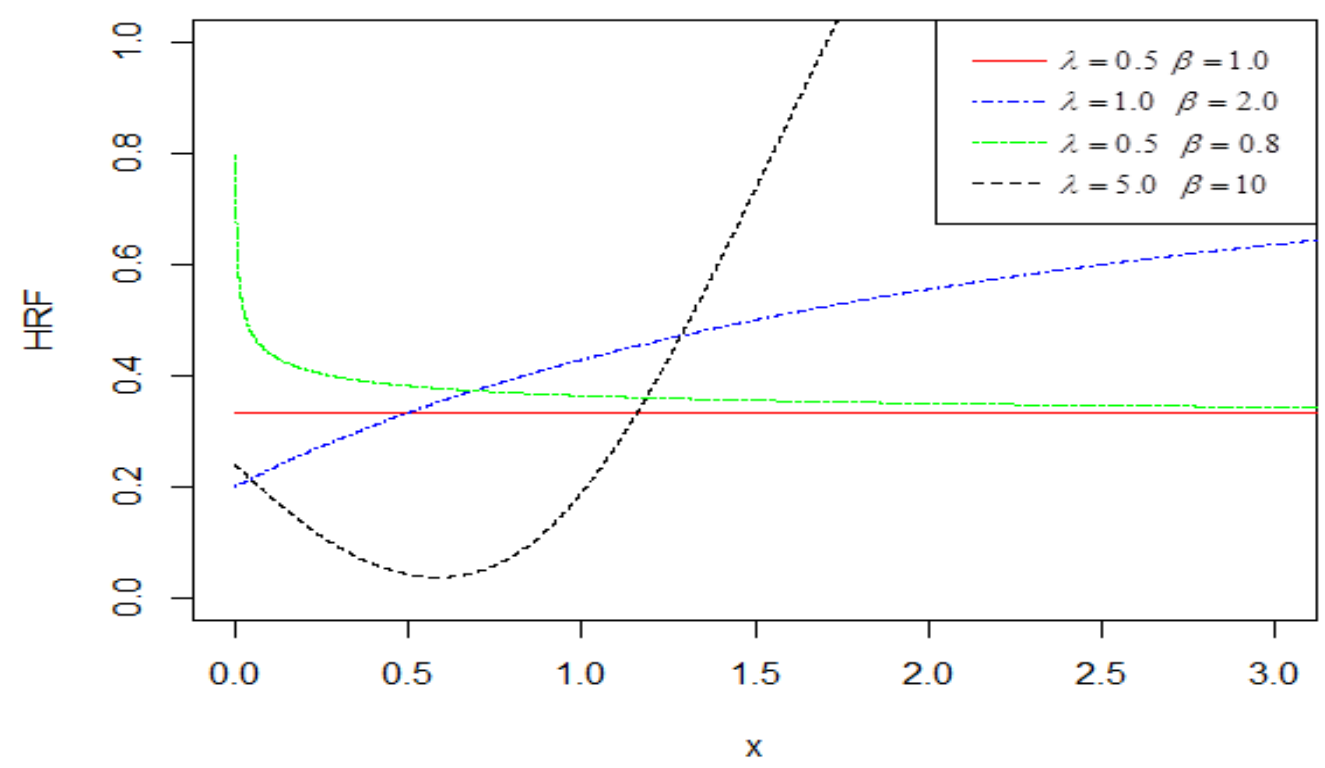

Figure 2. The Hazard Rate Function of NMEG distribution

Hence, the superiority and flexibility of the proposed distribution over some existing distribution which includes but not limited to Exponential, Lindley and Gamma distributions.

\section{STATISTICAL PROPERTIES OF THE NMEG DISTRIBUTION}

\subsection{Moment and Related Measure}

The $\mathrm{k}^{\text {th }}$ raw moment of a continuous random variable $Y$ denoted by $\mu_{k}^{\prime}$ is defined as

$$
\mu_{k}^{\prime}=E\left(X^{k}\right)=\int_{-\infty}^{\infty} y^{k} f(y) d y .
$$

Upon substituting (7) into (13), the $\mathrm{k}^{\text {th }}$ moment of the NMEG distribution is given by:

$$
\begin{aligned}
\mu_{k}^{\prime} & =E\left(X^{k}\right)=\frac{\lambda}{1+\beta} \int_{0}^{\infty} y^{k}\left\{1+\frac{\beta(\lambda y)^{\beta-1}}{\Gamma(\beta)}\right\} e^{-\lambda y} d x, \\
& =\frac{1}{1+\beta}\left\{\lambda \int_{0}^{\infty} y^{k} e^{-\lambda y} d y+\frac{\beta \lambda^{\beta}}{\Gamma(\beta)} \int_{0}^{\infty} y^{k+\beta-1} e^{-\lambda y} d y\right\} .
\end{aligned}
$$

Substituting $Z=\lambda y$, we have

$$
\mu_{k}^{\prime}=\frac{1}{1+\beta}\left\{\lambda \int_{0}^{\infty} \frac{z^{k}}{\lambda^{k+1}} e^{-z} d z+\frac{\beta \lambda^{\beta}}{\Gamma(\beta)} \int_{0}^{\infty}{\frac{z}{\lambda^{k+\beta}}}^{k+\beta-1} e^{-z} d z\right\}
$$




$$
\begin{aligned}
& =\frac{1}{1+\beta}\left\{\lambda \frac{\Gamma(k+\beta)}{\lambda^{k+1}}+\frac{\beta \lambda^{\beta}}{\Gamma(\beta)} \frac{\Gamma(k+\beta-1)}{\lambda^{k+1}}\right\}, \\
& =\frac{1}{\lambda^{k}(1+\beta)}\left\{k !+\frac{\beta \Gamma(k+\beta)}{\Gamma(\beta)}\right\} .
\end{aligned}
$$

It becomes easy to obtain the first four raw moment of the NMEG distribution as follows $\mu=\mu_{1}^{\prime}=\frac{1}{\lambda(1+\beta)}\left\{1+\beta^{2}\right\}$.

Table 1 shows the values of mean $(\mu)$, standard deviation $(\sigma)$, coefficient of variation $(\xi)$, measure of skewness $\left(S_{k}\right)$ and measure of kurtosis $\left(K_{s}\right)$ for some selected parameter values.

\begin{tabular}{|c|c|c|c|c|c|c|}
\hline \multicolumn{2}{|c|}{ Parameters } & \multirow[b]{2}{*}{$\mu$} & \multirow[b]{2}{*}{$\sigma$} & \multirow[b]{2}{*}{$\xi$} & \multirow[b]{2}{*}{$S_{k}$} & \multirow[b]{2}{*}{$K_{s}$} \\
\hline$\beta$ & $\lambda$ & & & & & \\
\hline \multirow[t]{5}{*}{0.2} & 0.2 & 4.3333 & 4.8872 & 1.1279 & -0.2477 & 8.7163 \\
\hline & 0.5 & 1.7333 & 1.9550 & 1.1279 & 0.2347 & 7.0053 \\
\hline & 1.4 & 0.6190 & 0.6982 & 1.1279 & 1.6821 & 1.8722 \\
\hline & 1.7 & 0.5098 & 0.5750 & 1.1279 & 2.1646 & 0.1612 \\
\hline & 2.0 & 0.4333 & 0.4887 & 1.1279 & 2.6470 & -1.5498 \\
\hline \multirow[t]{5}{*}{0.5} & 0.2 & 4.1667 & 4.7140 & 1.1313 & -0.1436 & 8.8974 \\
\hline & 0.5 & 1.6667 & 1.8856 & 1.1313 & 0.3535 & 7.1396 \\
\hline & 1.4 & 0.5952 & 0.6734 & 1.1313 & 1.8451 & 1.8662 \\
\hline & 1.7 & 0.4901 & 0.5545 & 1.1313 & 2.3422 & 0.1083 \\
\hline & 2.0 & 0.4167 & 0.4714 & 1.1313 & 2.8394 & -16494 \\
\hline \multirow[t]{5}{*}{1.4} & 0.2 & 6.1667 & 5.6396 & 0.9145 & -1.0578 & 12.4257 \\
\hline & 0.5 & 2.4667 & 2.2558 & 0.9145 & -.04218 & 9.6439 \\
\hline & 1.4 & 0.8809 & 0.8056 & 0.9145 & 1.4861 & 1.2985 \\
\hline & 1.7 & 0.7254 & 0.6634 & 0.9145 & 2.1221 & -1.4832 \\
\hline & 2.0 & 0.6167 & 0.5639 & 0.9145 & 2.7582 & -4.2650 \\
\hline \multirow[t]{5}{*}{1.7} & 0.2 & 7.2037 & 6.2349 & 0.8655 & -1.3858 & 14.3388 \\
\hline & 0.5 & 2.8814 & 2.4939 & 0.8655 & -0.7435 & 11.3705 \\
\hline & 1.4 & 1.0291 & 0.8907 & 0.8655 & 1.1832 & 2.4657 \\
\hline & 1.7 & 0.8474 & 0.7335 & 0.8655 & 1.8255 & -0.5025 \\
\hline & 2.0 & 0.7203 & 0.6234 & 0.8655 & 2.4678 & -3.4708 \\
\hline \multirow[t]{5}{*}{2.0} & 0.2 & 8.3333 & 6.8718 & 0.8246 & -1.6977 & 16.5674 \\
\hline & 0.5 & 3.3333 & 2.7487 & 0.8246 & -1.0557 & 13.4532 \\
\hline & 1.4 & 1.1904 & 0.9816 & 0.8246 & 0.8702 & 4.1107 \\
\hline & 1.7 & 0.9803 & 0.8084 & 0.8246 & 1.5122 & 0.9965 \\
\hline & 2.0 & 0.8333 & 0.6871 & 0.8246 & 2.1542 & -2.1176 \\
\hline
\end{tabular}

Table 1. Table of Mean, Standard Deviation, Coefficient of Variation, Skewness and Kurtosis

Table 1 shows that the NMEG distribution can be positively skewed or negatively skewed for some selected parameter values. Also, the kurtosis can be Leptokurtic (heavy tailed) distribution for $K_{s}>3$ or 
Platykurtic (light tailed) distribution for $K_{s}<3$. The negative kurtosis belong to the platykurtic distribution class with a broad shoulder. This means that the peak is broader and wider than the normal distribution. See Peter [19] for more details on negative kurtosis.

$$
\begin{aligned}
& \mu_{2}^{\prime}=\frac{1}{\lambda^{2}(1+\beta)}\left\{2+\beta^{2}(1+\beta)\right\} . \\
& \mu_{3}^{\prime}=\frac{1}{\lambda^{3}(1+\beta)}\left\{6+\beta^{2}(1+\beta)(2+\beta)\right\} . \\
& \mu_{4}^{\prime}=\frac{1}{\lambda^{4}(1+\beta)}\left\{24+\beta^{2}(1+\beta)(2+\beta)(1+\beta)\right\} .
\end{aligned}
$$

Also, the central moment is given by the expression

$$
\begin{aligned}
\mu_{k}= & E(X-\mu)^{k}=E\left\{\sum_{i=1}^{k}\left(\begin{array}{l}
k \\
i
\end{array}\right) X^{k-i}(-\mu)^{i}\right\} \\
& =\sum_{i=1}^{k}\left(\begin{array}{l}
k \\
i
\end{array}\right)(-1)^{i} \mu_{K-i}^{\prime} \mu^{i} .
\end{aligned}
$$

The 2nd, 3rd and 4th central moments can be obtained as

$$
\mu_{2}=\mu_{2}^{\prime}-\mu^{2}, \mu_{3}=\mu_{3}^{\prime}-3 \mu_{2}^{\prime} \mu+2 \mu^{3} \text { and } \mu_{4}=\mu_{4}^{\prime}-4 \mu_{3}^{\prime} \mu+6 \mu_{2}^{\prime} \mu-3 \mu^{4}
$$

The mean $(\mu)$, variance $\left(\sigma^{2}\right)$, coefficient of variation $(\xi)$, skewness $\left(S_{k}\right)$ and $\operatorname{kurtosis}\left(K_{s}\right)$ can be obtained respectively using the expression:

$$
\mu=\mu_{1}^{\prime}, \sigma^{2}=\mu_{2}^{\prime}-\mu^{2}, \xi=\frac{\sigma}{\mu}, S_{k}=\frac{\mu_{3}}{\left(\mu_{2}\right)^{\frac{3}{2}}}, K_{s}=\frac{\mu_{4}}{\left(\mu_{2}\right)^{2}} .
$$

\subsection{Moment Generating Function}

The moment generating function of a random variable $y$, with pdf, $f(y)$ is given by;

$$
M_{Y}(z)=E\left(e^{z y}\right)=\int_{-\infty}^{\infty} e^{z y} f(y) d y .
$$

Thus, we have the moment generating function of the NMEG distribution as

$$
\begin{aligned}
M_{Y}(y)=E\left(e^{z y}\right) & =\frac{1}{1+\beta} \int_{0}^{\infty} e^{z y}\left\{\lambda+\frac{\beta \lambda(\lambda y)^{\beta-1}}{\Gamma(\beta)}\right\} e^{-\lambda y} d y \\
& =\frac{1}{1+\beta}\left\{\lambda \int_{0}^{\infty} e^{-(\lambda-z) y} d y+\frac{\beta \lambda^{\beta}}{\Gamma(\beta)} \int_{0}^{\infty} y e^{-(\lambda-z) y} d y\right\} \\
& =\frac{1}{1+\beta}\left\{\lambda \frac{1}{\lambda-z}+\frac{\beta \lambda^{\beta}}{\Gamma(\beta)} \frac{\Gamma(\beta)}{(\lambda-z)^{\beta}}\right\} \\
& =\frac{1}{1+\beta}\left\{\frac{\lambda}{\lambda-z}+\frac{\beta \lambda^{\beta}}{(\lambda-z)^{\beta}}\right\} .
\end{aligned}
$$




\subsection{Quantile Function}

The quantile function of a probability distribution with CDF, $F(x)$ is defined by $q=F^{-1}\left(x_{q}\right)$ where $0<q<1$. Hence, the quantile function of the new mixture of exponential-gamma (NMEG) distribution is derived as follows

$$
\begin{aligned}
& q=\frac{1}{1+\beta}\left(\frac{\beta \gamma\left(\beta, \lambda y_{q}\right)}{\Gamma(\beta)}+1-e^{-\lambda y_{q}}\right) \\
& q(1+\beta)=\frac{\beta \gamma\left(\beta, \lambda y_{q}\right)}{\Gamma(\beta)}+G\left(y_{q}\right),
\end{aligned}
$$

where $G(y)$ is the CDF of exponential distribution which is one of the mixture density of the NMEG distribution

$$
\gamma\left(\beta, \lambda y_{q}\right)=q(1+\beta) \Gamma(\beta)-\frac{\Gamma(\beta)}{\beta G^{-1}\left(y_{q}\right)} .
$$

Using the idea of Samir, et al. [7], we derive the quantile function as follows

$$
\lambda y_{q}=\gamma^{-1}\left(\beta, q(1+\beta) \Gamma(\beta)-\frac{\Gamma(\beta)}{\beta G^{-1}\left(y_{q}\right)}\right) .
$$

But $G^{-1}\left(y_{q}\right)=-\frac{\log (1-q)}{\lambda}$, which is the quantile function of the exponential distribution. Then the quantile function of the NMEG becomes

$$
Q_{q}=F^{-1}\left(y_{q}\right)=\frac{1}{\lambda} \gamma^{-1}\left(\beta, q(1+\beta) \Gamma(\beta)-\frac{\lambda \Gamma(\beta)}{\beta \log (1-q)}\right) .
$$

Random samples can be generated from the NMEG distribution using (17). The $1^{\text {st }}, 2^{\text {nd }}$ and $3^{\text {rd }}$ quartiles of the NMEG distribution are given by

$$
\begin{aligned}
& Q_{1}=F^{-1}\left(\frac{1}{4}\right)=\frac{1}{\lambda} \gamma^{-1}\left(\beta, \frac{1}{4}(1+\beta) \Gamma(\beta)-\frac{\lambda \Gamma(\beta)}{\beta \log \left(\frac{3}{4}\right)}\right), \\
& Q_{2}=F^{-1}\left(\frac{1}{2}\right)=\frac{1}{\lambda} \gamma^{-1}\left(\beta, \frac{1}{2}(1+\beta) \Gamma(\beta)-\frac{\lambda \Gamma(\beta)}{\beta \log \left(\frac{1}{2}\right)}\right), \\
& Q_{3}=F^{-1}\left(\frac{3}{4}\right)=\frac{1}{\lambda} \gamma^{-1}\left(\beta, \frac{3}{4}(1+\beta) \Gamma(\beta)-\frac{\lambda \Gamma(\beta)}{\beta \log \left(\frac{1}{4}\right)}\right) .
\end{aligned}
$$




\subsection{Distribution of Order Statistics}

Let $Y_{1}, Y_{2}, \ldots, Y_{n}$ be a random sample of size $\mathrm{n}$ from the NMEG distribution whose pdf and CDF are given in (7) and (8) respectively, then the pdf of the $r$ th order statistics $Y_{r: n}, r=1,2,3, \ldots, n$ denoted by $f_{r: n}(y)$, for $1 \leq y \leq n$

is defined as

$$
\begin{aligned}
& f_{r: n}(y ; \Phi)=\frac{n !}{(r-1) !(n-r) !} f(y) F^{r-1}(y)[1-F(y)]^{n-r}, \\
& =\frac{n !}{(r-1) !(n-r) !} \sum_{i=0}^{n-r}\left(\begin{array}{c}
n-r \\
i
\end{array}\right)(-1)^{i} f(y)[F(y)]^{r+i-1},
\end{aligned}
$$

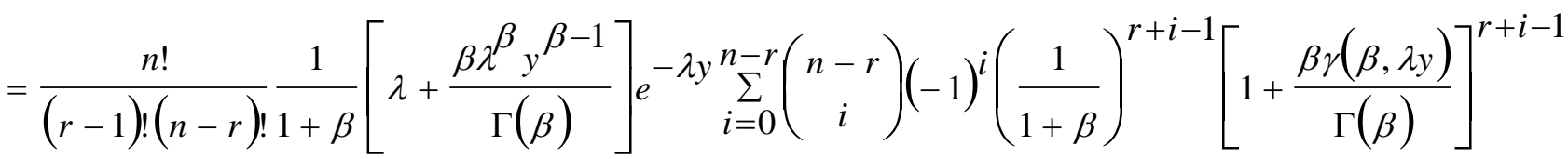

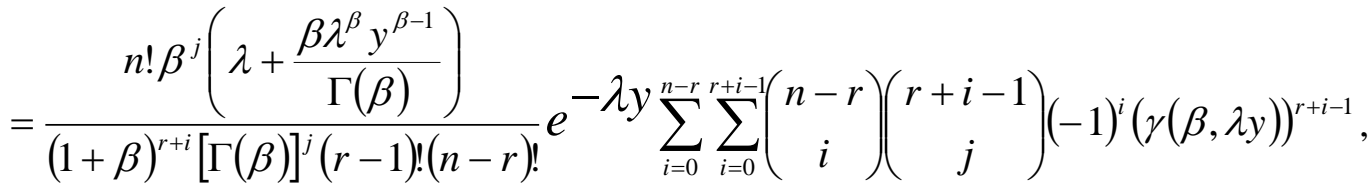

$$
\begin{aligned}
& =\frac{n ! \beta^{j+k}\left(\lambda+\frac{\beta \lambda^{\beta} y^{\beta-1}}{\Gamma(\beta)}\right)}{(1+\beta)^{r+i}[\Gamma(\beta)]^{j}(r-1) !(n-r) !} \sum_{k=0}^{\infty} \sum_{i=0}^{n-r} \sum_{i=0}^{r+i-1}\left(\begin{array}{c}
n-r \\
i
\end{array}\right)\left(\begin{array}{c}
r+i-1 \\
j
\end{array}\right)(-1)^{i}(\gamma(\beta, \lambda y))^{r+i-1} \frac{y^{k}}{k !} .
\end{aligned}
$$

\subsection{Renyi Entropy}

Entropy is an important concept to measure the quantity of uncertainty in relation to a random variable say $Y$. Renyi [20] defined the entropy of a random variable $Y$ with pdf $f(y)$ as

$$
J_{R}(s)=\frac{1}{1-s} \log \int_{-\infty}^{\infty}[f(y)]^{s} d y, \quad s>0, s \neq 1 .
$$

Then, using (7), the NMEG distribution becomes

where

$$
J_{R}(s)=\frac{1}{1-s} \log \Phi(s),
$$

$$
\begin{aligned}
& \Phi(s)=\left(\frac{1}{1+\beta}\right)^{s} \int_{0}^{\infty}\left[\lambda+\frac{\beta \lambda^{\beta} y^{\beta-1}}{\Gamma(\beta)}\right]^{s} e^{-s \lambda y} d y \\
& =\left(\frac{1}{1+\beta}\right)^{s} \sum_{i=0}^{\infty}\left(\begin{array}{l}
s \\
i
\end{array}\right) \lambda^{s-i}\left(\frac{\beta \lambda^{\beta}}{\Gamma(\beta)}\right)^{i} \int_{0}^{\infty} y^{(\beta-1) i} e^{-s \lambda y} d y .
\end{aligned}
$$


Using the transformation $u=s \lambda y$, we have

$$
\begin{aligned}
& \Phi(s)=\left(\frac{1}{1+\beta}\right)^{s} \sum_{i=0}^{\infty}\left(\begin{array}{l}
s \\
i
\end{array}\right) \lambda^{s-i}\left(\frac{\beta \lambda^{\beta}}{\Gamma(\beta)}\right)^{i} \int_{0}^{\infty} \frac{u^{(\beta-1) i}}{(s \lambda)^{(\beta-1) i}} e^{-u} d u \\
& =\left(\frac{1}{1+\beta}\right)^{s} \sum_{i=0}^{\infty}\left(\begin{array}{l}
s \\
i
\end{array}\right) \lambda^{s-i}\left(\frac{\beta \lambda^{\beta}}{\Gamma(\beta)}\right)^{i} \frac{((\beta-1) i+1)}{(s \lambda)^{(\beta-1) i+1}} \\
& =\left(\frac{1}{1+\beta}\right)^{s} \sum_{i=0}^{\infty}\left(\begin{array}{l}
s \\
i
\end{array}\right) \frac{\beta^{i}}{[\Gamma(\beta)]^{i}} \frac{\lambda^{s+i} \Gamma((\beta-1) i+1)}{s^{(\beta-1) i+1}}
\end{aligned}
$$

Hence,

$$
J_{R}(s)=\frac{1}{1-s} \log \left\{\left(\frac{1}{1+\beta}\right)^{s} \sum_{i=0}^{\infty}\left(\begin{array}{l}
s \\
i
\end{array}\right) \frac{\beta^{i}}{[\Gamma(\beta)]^{i}} \frac{\lambda^{s+i} \Gamma((\beta-1) i+1)}{s^{(\beta-1) i+1}}\right\} .
$$

\section{MAXIMUM LIKELIHOOD ESTIMATION}

Let $y_{1,} y_{2}, \ldots, y_{n}$ be a random sample from NMEG distribution, then the log-likelihood function, $\ell(y ; \Phi)$ is defined by

$$
\begin{aligned}
\ell(y ; \Phi) & =\sum_{i=1}^{n} \log \left\{\frac{1}{1+\beta}\left[\lambda+\frac{\beta \lambda^{\beta} y_{i}^{\beta-1}}{\Gamma(\beta)}\right] e^{-\lambda y_{i}}\right\} \\
& =-n \ln (1+\beta)+\sum_{i=1}^{n} \ln \left[\lambda \Gamma(\beta)+\beta \lambda^{\beta} y_{i}^{\beta-1}\right]-n \ln \Gamma(\beta)-\lambda \sum_{i=1}^{n} y_{i} .
\end{aligned}
$$

The partial derivatives of (20) with respect to the parameters $\lambda$ and $\beta$ are:

$$
\begin{aligned}
& \frac{\partial \ell}{\partial \lambda}=\sum_{i=1}^{n} \frac{\Gamma(\beta)+\beta^{2}(\lambda y)^{\beta-1}}{\lambda \Gamma(\beta)+\beta \lambda(\lambda y)^{\beta-1}}-\sum_{i=1}^{n} y_{i} \\
& \frac{\partial \ell}{\partial \beta}=-\frac{n}{1+\beta}-\frac{n \Gamma^{\prime}(\beta)}{\Gamma(\beta)}+\sum_{i=1}^{n} \frac{\lambda \Gamma^{\prime}(\beta)+(\lambda y)^{\beta-1}\left[\lambda+\ln \left(\lambda y_{i}\right)^{\beta-1}\right]}{\lambda \Gamma(\beta)+\beta \lambda\left(\lambda y_{i}\right)^{\beta-1}} .
\end{aligned}
$$

The MLEs $(\hat{\lambda}, \hat{\beta})$ of $(\lambda, \beta)$, can be obtained by solving $\frac{\partial \ell}{\partial \lambda}=0$ and $\frac{\partial \ell}{\partial \beta}=0$ simultaneously. This is called the score function given by

$$
U(\Phi)=\left(\frac{\partial \ell}{\partial \lambda}, \frac{\partial \ell}{\partial \beta}\right)^{T}=0
$$

It is clear that equations (22)-(24) has no explicit analytical solution, hence, it can be solved numerically using Newton-Raphson iterative method which a powerful technique for solving nonlinear system of equations. This numerical estimates of $\hat{\Phi}=(\hat{\lambda}, \hat{\beta})$ are easily obtained using $\mathrm{R}$ statistical software.

For the interval estimate of the distribution parameters $\Phi=(\lambda, \beta)$, we obtain the $2 \times 2$ observed information matrix whose elements are the second derivatives of the log-likelihood function as 


$$
I(\Phi)=-E\left(\begin{array}{ll}
\frac{\partial^{2} \ell}{\partial \lambda^{2}} & \frac{\partial^{2} \ell}{\partial \lambda \partial \beta} \\
\frac{\partial^{2} \ell}{\partial \beta \partial \lambda} & \frac{\partial^{2} \ell}{\partial \beta^{2}}
\end{array}\right)_{\lambda=\hat{\lambda}, \beta=\hat{\beta}}
$$

The approximate $100(1-\alpha) \%$ two sided confidence intervals for $\lambda$ and $\beta$ are respectively given by

$$
\hat{\lambda} \pm Z_{\alpha / 2} \sqrt{I_{\lambda \lambda}^{-1}(\hat{\Phi})} \text { and } \hat{\beta} \pm Z_{\alpha / 2} \sqrt{I_{\beta \beta}^{-1}(\hat{\Phi})}
$$

where $I_{\lambda \lambda}^{-1}(\hat{\Phi})$ and $I_{\beta \beta}^{-1}(\hat{\Phi})$ are the diagonals elements of $I_{n}^{-1}(\hat{\Phi})=(n I \hat{\Phi})^{-1}$ and $Z_{\alpha / 2}$ is the upper $(\alpha / 2)^{t h}$ percentile of a standard normal distribution.

\section{SIMULATION STUDY}

In this section, we carry out simulation study to examine the performance and accuracy of the Maximum Likelihood Estimates (MLEs) of the NMEG distribution. In each simulation, 10,000 samples of sizes $n=50,75,100,150$ and 200 were generated for different values of the parameters $\lambda$ and $\beta$ using the quantile function of the NMEG distribution in equation (15). For each sample, the MLEs are obtained, these are used to compute values of the following quantities with the help of $\mathrm{R}$ package.

(i) $\quad$ Average bias $=\frac{1}{N} \sum_{i=1}^{N}(\hat{\Phi}-\Phi)$,

(ii) Mean Square Error (MSE) $=\frac{1}{N} \sum_{i=1}^{N}(\hat{\Phi}-\Phi)^{2}$,

(iii) Coverage Probability (CP) of $95 \%$ confidence intervals of the parameters $\Phi=\lambda, \beta$ and

(iv) Average Width (AW) of $95 \%$ confidence intervals of the parameters $\Phi=\lambda, \beta$.

Table 2 shows the values of the four quantities: Av.Bias, MSE, CP and AW of the parameters $\lambda$ and $\beta$ for different sample sizes $n=50,75,100,150$ and 200. From the results, we observed that as the sample size $\mathrm{n}$ increases, the value of average bias and MSE decreases. It is also clear to see from Table 2 that for all the parameters, the coverage probabilities of the confidence intervals are also close to the nominal level of $95 \%$ and the average confidence widths decreases as the sample size increases. Hence, we can use the MLE's and their asymptotic results for estimating and constructing confidence intervals for a reasonable sample size. 
Table 2. Average Bias, (Av.Bias), Mean Square Error, (MSE), Coverage Probability, (CP), and Average Width, (AW) of 10,000 MLEs of the new gamma mixture (NMEG) for different fixed values of the parameters

\begin{tabular}{|c|c|c|c|c|c|c|c|c|c|}
\hline \multirow[t]{2}{*}{ Param. } & \multirow[t]{2}{*}{$n$} & \multicolumn{4}{|c|}{ Av.Bias } & \multicolumn{4}{|c|}{ Av.Bias } \\
\hline & & $(\hat{\lambda})$ & $\operatorname{MSE}(\hat{\lambda})$ & $C P(\hat{\lambda})$ & $A W(\hat{\lambda})$ & $(\hat{\beta})$ & $\operatorname{MSE}(\hat{\beta})$ & $C P(\hat{\beta})$ & $A W(\hat{\beta})$ \\
\hline \multirow{5}{*}{$\lambda=0.5, \beta=1.5$} & 50 & 0.1273 & 0.0513 & 0.966 & 0.7099 & 0.2525 & 0.4163 & 0.934 & 2.3562 \\
\hline & 75 & 0.1162 & 0.0400 & 0.928 & 0.5805 & 0.2451 & 0.3565 & 0.904 & 1.9647 \\
\hline & 100 & 0.0920 & 0.0252 & 0.934 & 0.4900 & 0.1604 & 0.2293 & 0.912 & 1.6835 \\
\hline & 150 & 0.0792 & 0.0170 & 0.934 & 0.3982 & 0.1312 & 0.1527 & 0.922 & 1.3883 \\
\hline & 200 & 0.0278 & 0.0101 & 0.922 & 0.3441 & 0.0664 & 0.1181 & 0.908 & 1.1960 \\
\hline \multirow{5}{*}{$\lambda=1.0, \beta=2.0$} & 50 & 0.1017 & 0.1419 & 0.908 & 1.3328 & 0.1563 & 0.5559 & 0.898 & 2.7089 \\
\hline & 75 & 0.0950 & 0.1000 & 0.926 & 1.1458 & 0.1432 & 0.3807 & 0.916 & 2.3230 \\
\hline & 100 & 0.0688 & 0.0767 & 0.908 & 0.9721 & 0.0921 & 0.3009 & 0.896 & 1.9842 \\
\hline & 150 & 0.0356 & 0.0459 & 0.924 & 0.7999 & 0.0448 & 0.1884 & 0.926 & 1.6554 \\
\hline & 200 & 0.0274 & 0.0376 & 0.910 & 0.6894 & 0.0446 & 0.1623 & 0.906 & 1.4342 \\
\hline \multirow{5}{*}{$\lambda=1.5, \beta=2.5$} & 50 & 0.1204 & 0.2655 & 0.908 & 1.8729 & 0.1308 & 0.6657 & 0.920 & 3.0029 \\
\hline & 75 & 0.0836 & 0.1550 & 0.942 & 1.5517 & 0.0957 & 0.3763 & 0.932 & 2.5028 \\
\hline & 100 & 0.0623 & 0.1233 & 0.928 & 1.3422 & 0.0759 & 0.3055 & 0.946 & 2.1803 \\
\hline & 150 & 0.0623 & 0.0922 & 0.934 & 1.1156 & 0.0751 & 0.2336 & 0.932 & 1.8063 \\
\hline & 200 & 0.0248 & 0.0617 & 0.940 & 0.9488 & 0.0336 & 0.1524 & 0.956 & 1.5441 \\
\hline
\end{tabular}

\section{REAL DATA APPLICATION}

In this section, we show the applicability of the NMEG distribution by considering real life data sets which shows that the NMEG out performs the Gamma (Gam.), Lindley (Lind.) and Exponential (Exp.) distributions. The pdf of these distributions are given below as:

(i) Gamma Distribution:

$$
f_{g}(y)=\frac{\lambda(\lambda y)^{\beta-1}}{\Gamma(\beta)} e^{-\lambda y}, \quad y>0, \beta, \lambda>0 .
$$

(ii) Lindley Distribution:

$$
f_{L}(x)=\frac{\lambda^{2}(1+y) e^{-\lambda y}}{1+\lambda}, \quad y>0, \lambda>0 .
$$

(iii) Exponential Distribution:

$$
f_{E}(y)=\lambda e^{-\lambda x}, \quad y>0, \lambda>0 .
$$

Also, for comparison of statistical model, we used goodness of fit criteria namely: $\log$-likelihood $(\ell)$ and Akaike Information Criteria $(A I C)$, as well as goodness of fit statistics namely: Kolmogorov- Smirnov $(K-S)$ statistics, Cramer-von Mises $(C-V M)$ statistics and Anderson-Darling $(A-D)$ statistics to choose the best possible model for the fitted data sets. In general, the smaller the value of the goodness of fit criteria/statistics, the better the fit to the data set. These computations are executed in R-Package environment.

\subsection{Data Set 1}

The given data represents the breaking stress of carbon fibers (in Gba) as observed and reported by Nichols and Padgett [21]: 


$\begin{array}{llllllllllll}3.70, & 2.74, & 2.73, & 2.50, & 3.60, & 3.11, & 3.27, & 2.87, & 1.47, & 3.11, & 3.56, & 4.42, \\ 2.41, & 3.19, & 3.22, & 1.69, & 3.28, & 3.09, & 1.87, & 3.15, & 4.90, & 1.57, & 2.67, & 2.93, \\ 3.22, & 3.39, & 2.81, & 4.20, & 3.33, & 2.55, & 3.31, & 3.31, & 2.85, & 1.25, & 4.38, & 1.84, \\ 0.39, & 3.68, & 2.48, & 0.85, & 1.61, & 2.79, & 4.70, & 2.03, & 1.89, & 2.88, & 2.82, & 2.05, \\ 3.65, & 3.75, & 2.43, & 2.95, & 2.97, & 3.39, & 2.96, & 2.35, & 2.55, & 2.59, & 2.03, & 1.61, \\ 2.12, & 3.15, & 1.08, & 2.56, & 1.80, & 2.53 & & & & & & \end{array}$

Table 3. Summary Statistics for the Data Set 1

\begin{tabular}{|c|c|c|c|c|c|c|}
\hline Distr. & Estimates & $\ell$ & $A I C$ & $K-S$ & $C-V M$ & $A-D$ \\
\hline NGM & $\begin{array}{c}\lambda=3.90531 \\
\beta=11.07067\end{array}$ & -90.0958 & 184.1917 & 0.118516 & 0.213428 & 1.150478 \\
\hline GAM & $\begin{array}{l}\lambda=2.71397 \\
\beta=7.48908\end{array}$ & -91.1675 & 186.3351 & 0.132869 & 0.246366 & 1.311358 \\
\hline $\begin{array}{l}\text { LIND } \\
\text { EXP }\end{array}$ & $\begin{array}{c}\lambda=0.59025 \\
\lambda=0.36237\end{array}$ & $\begin{array}{l}-122.3841 \\
-132.9944\end{array}$ & $\begin{array}{l}246.7681 \\
267.9887\end{array}$ & $\begin{array}{l}0.297702 \\
0.358114\end{array}$ & $\begin{array}{l}2.091412 \\
2.871046\end{array}$ & $\begin{array}{l}10.692207 \\
14.034266\end{array}$ \\
\hline
\end{tabular}

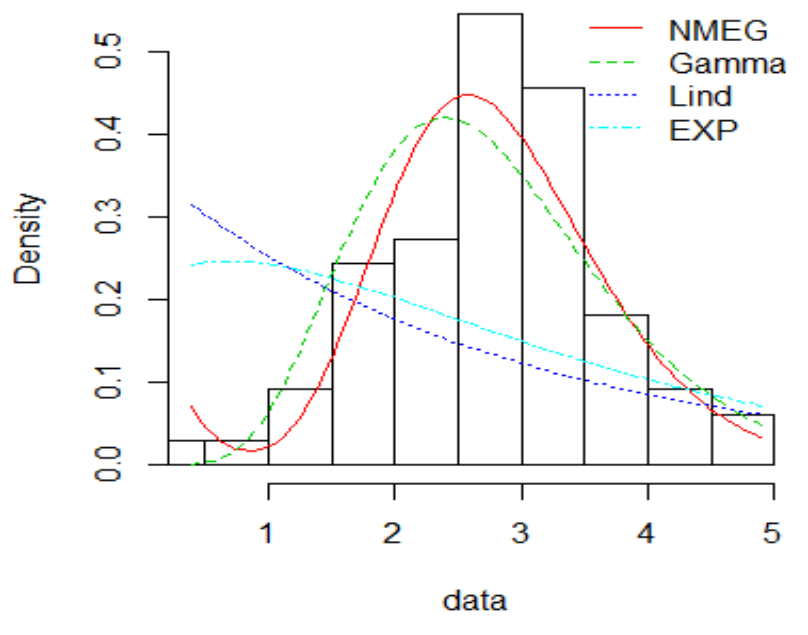

(a) Estimated pdfs

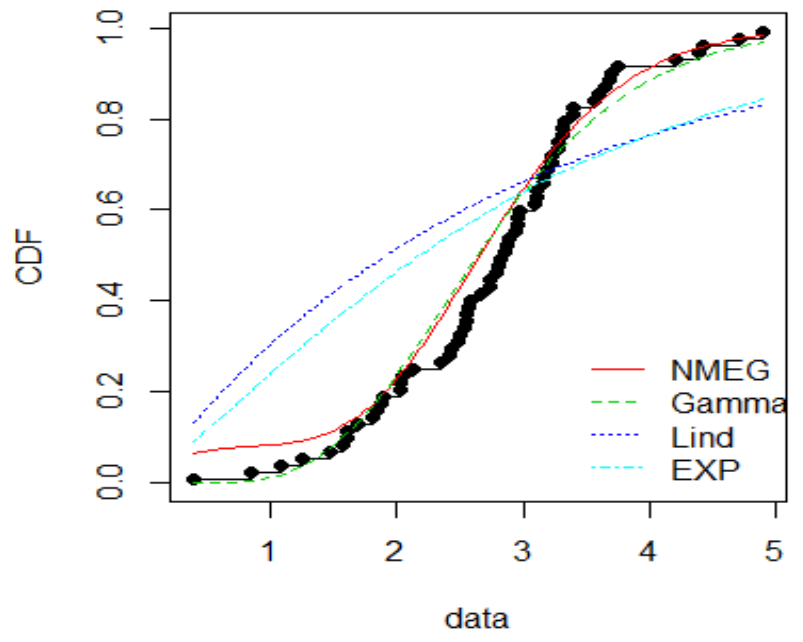

(b) Estimated CDFs

Figure 3. The Plots (a) and (b) of the Estimated Density and Cumulative Distributions Functions respectively for the Data Set 1

\subsection{Data Set 2}

This real data set represents the survival times of 121 patients with breast cancer obtained from a large hospital in a period from 1929 to 1938 taken from Lee [22]:

0.3, 0.3, 4.0, 5.0, 5.6, 6.2, 6.3, 6.6, 6.8, 7.4, 7.5, 8.4, 8.4, 10.3,11.0, 11.8, 12.2, 12.3, 13.5, 14.4, 14.4, 14.8, $15.5,15.7,16.2,16.3,16.5,16.8,17.2,17.3,17.5,17.9,19.8,20.4,20.9,21.0,21.0,21.1,23.0,23.4,23.6$, 24.0, 24.0, 27.9, 28.2, 29.1, 30.0, 31.0, 31.0, 32.0, 35.0, 35.0, 37.0, 37.0, 37.0, 38.0, 38.0, 38.0, 39.0, 39.0, 40.0, 40.0, 40.0, 41.0, 41.0, 41.0, 42.0, 43.0, 43.0, 43.0, 44.0, 45.0, 45.0, 46.0, 46.0, 47.0, 48.0, 49.0, 51.0, 51.0, 51.0, 52.0, 54.0, 55.0, 56.0, 57.0, 58.0, 59.0, 60.0, 60.0, 60.0, 61.0, 62.0, 65.0, 65.0, 67.0, 67.0, 68.0, 69.0, 78.0, 80.0,83.0, 88.0, 89.0, 90.0, 93.0, 96.0, 103.0, 105.0, 109.0, 109.0, 111.0, 115.0, 117.0, 125.0, 126.0, 127.0, 129.0, 129.0, 139.0, 154 . 
Table 4. Summary Statistics for the Data Set 2

\begin{tabular}{llccccc}
\hline Distr. & Estimates & $\ell$ & AIC & $K-S$ & $C-V$ & $A-D$ \\
\hline NGM & $\lambda=0.04122$ & -579.1782 & 1162.356 & 0.0617 & 0.0577 & 0.4246 \\
& $\beta=2.28889$ & & & & & \\
GAM & $\lambda=0.03230$ & -579.8047 & 1163.609 & 0.0762 & 0.0611 & 0.4617 \\
& $\beta=1.49622$ & & & & & \\
LIND & $\lambda=0.02159$ & -585.1278 & 1172256 & 0.1205 & 0.4605 & 2.6986 \\
EXP & $\lambda=0.04229$ & -580.4314 & 1162.863 & 0.0744 & 0.1127 & 0.9428 \\
\hline
\end{tabular}

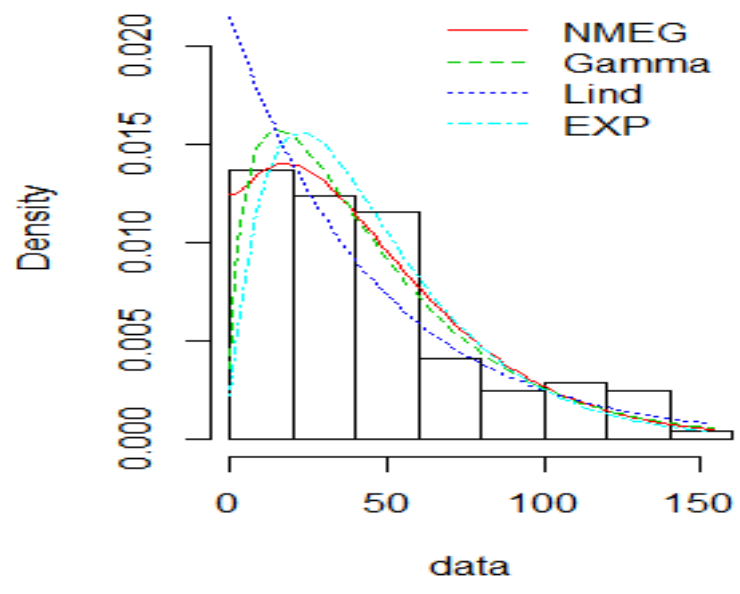

(a) Estimated pdfs

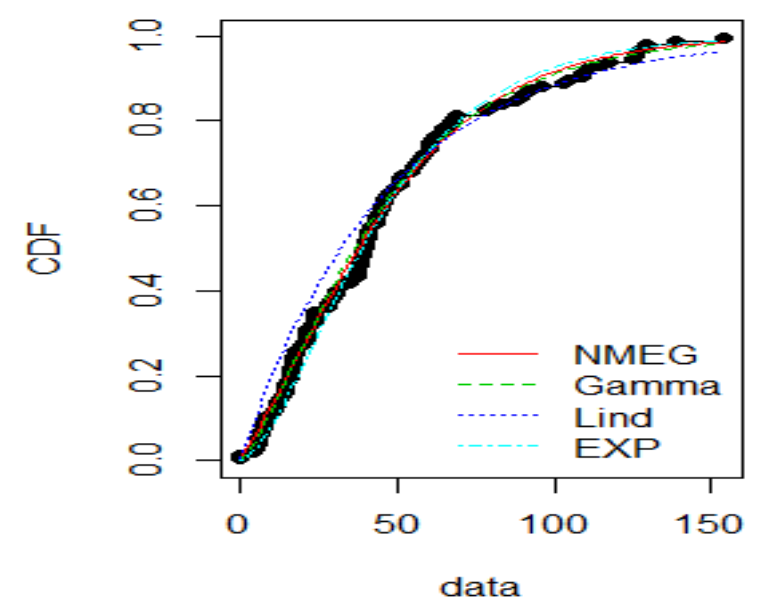

(b) Estimated CDFs

Figure 4. The Plots (a) and (b) of the Estimated Density and Cumulative Distributions Functions respectively for the Data Set 2

\subsection{Discussion of Result}

The values of the model comparison tools: $\log$-likelihood $(\ell)$, Akaike Information Criteria $(A I C)$, Kolmogorov-Smirnov $(K-S)$, Cramer-von Mises $(C-V M)$, and Anderson-Darling $(A-D)$ are shown in Tables 3 and 4 for data set 1 and 2 respectively. We can see from these tables that NMEG distribution have lower values of $\ell, A I C, K-S, C-V M$ and $A-D$ statistics amongst the fitted models namely: Gamma, Exponential and Lindley. This shows that the NMEG distribution outperforms the Exponential, Lindley and the classical Gamma distribution. Hence, NMEG distribution should be chosen as the most adequate model for these data sets.

Figures 3 and 4 displays the histogram of the data sets and the estimated pdfs and CDFs of the NMEG model and their competitive models.

\section{CONCLUSION}

In this paper, we introduced a new distribution called "New Gamma Mixture (NGM) Distribution". This is an extension of the classical gamma distribution for flexibility. The new distribution include the exponential and standardized Lindley distributions as sub-models. Some of the mathematical properties such as the raw moment, moment generating function, and the renyi entropy were studied. The maximum 
likelihood estimation (MLE) method was used to estimate parameters of the new distribution. An application of the NMEG distribution to a real lifetime data set demonstrates its superiority over the Exponential distribution, Lindley distribution and the classical Gamma distribution in modelling the lifetime data sets under study.

\section{CONFLICTS OF INTEREST}

No conflict of interest was declared by the authors.

\section{REFERENCES}

[1] Gupta, R.D., and Kundu, D., "Generalized Exponential Distributions", Australian and New Zealand Journal of Statistics, 41: $173-188$, (1999).

[2] Nadarajah, S. and Gupta, A.K., "A Generalized Gamma Distribution with Application to Drought Data", Mathematical Computation and Simulation, 74: 1-7, (2007).

[3] Mahdi, T., and Gupta, A.K., "A Generalization of The Gamma Distribution", Journal of Data Science, 11:403-414, (2013).

[4] Stacy, E.W., "A Generalization of the Gamma Distribution”, Annals Mathematical Statistics, 33: 1187-1192, (1962).

[5] Elbatal, I., Merovci, F., and Elgarhy, M., “A New Generalized Lindley Distribution”, Mathematical Theory and Modelling, 3(13):30-47, (2013).

[6] Gayan, W. L., and Mavis, P., "A Generalized Power Lindley Distribution with Applications", Asian Journal of Mathematics and Applications, ID: ama0169, 1-23, (2014).

[7] Samir, A., Darwish, D., and Ahmad, S., "Log-Gamma-Rayleigh Distribution: Properties and Applications", GU Journal of Science, 31(3): 967-983, (2018).

[8] Amini, M., MirMostafaee, S. M. T. K and Ahmeadi, J., "Log-Gamma-Generated Families of Distributions", Journal of Theoretical and Applied Statistics, 48 (4): 913-932, (2014)

[9] Lee, E. T., "Statistical Methods for Survival Data Analysis (2nd Edition)", John Wiley and Sons Inc., New York, USA, (1992).

[10] Nichols, M.D., and Padgett W.F., “A Bootstrap Control Chart for Weibull Percentiles” Qual. Reliab. Engng. Int. 22:141-151, 691, (2006).

[11] Alzaatreh, A., Lee, C., Famoye, F., "A New Method for Generating Families of Continuous Distributions", Metron, 71(1): 63-79, (2013).

[12] Eugene, N., Lee, C., Famoye, F., "Beta-Normal Distribution and its Applications", Communication in Statistics-Theory and Methods, 31(4): 497-512, (2002).

[13] Patil, G.P. and C.R. Rao, "Weighted Distributions and Size-Biased Sampling with Applications to Wildlife Populations and Human Families", Biometrics, 34: 179-189, (1978).

[14] Patill, G.P., C.R. Rao and M.V. Ratnaparkhi, "On Discrete Weighted Distributions and Their Use in Model Choice for Observed Data", Communication in Statistics- Theory and Methods, 15: 907918, (1986). 
[15] Resti, Y., Ismail N., and Jamaan S.H., "Estimation of Claim Cost Data Using Zero Adjusted Gamma and Inverse Gaussian Regression Models" Journal of Mathematical Statistics., 9: 186-192, (2013).

[16] Talangtam., T., and Sattayantham, P., "Fitting of finite mixture of lognormal distribution to motor insurance clams", Journal of Mathematical Statistics, 8: 49-56, (2012).

[17] Satsayamon S., and Winai B., "A New Family of Generalized Gamma Distribution and its Application", Journal of Mathematical Statistics, 10 (2): 211-220, (2014).

[18] Glasser, R., "Bathtub and Related Failure Rate Characterizations", Journal of American Statistical Association, 75: 667-672, (1980).

[19] Peter H. W., "Kurtosis as Peakedness", The American Statistician, 68:3, 191-195, (2014).

[20] Rényi, A., (1961). On measure of entropy and information. Proceedings of the $4^{\text {th }}$ Berkeley Symposium on Mathematical Statistics and Probability 1, University of California Press, Berkeley, page 547-561, (1961).

[21] Nichols, M. D., and Padgett, W. J., “A bootstrap control chart for Weibull percentiles”, Quality \& Reliability Engineering International, 22(2): 141-151, (2006).

[22] Lee, E. T., "Statistical Methods for Survival Data Analysis (2nd Edition)", John Wiley and Sons Inc., New York, USA, (1992). 